\title{
Article \\ Effects of COVID-19 Lockdown on Physical Activity, Sedentary Behavior, and Satisfaction with Life in Qatar: A Preliminary Study
}

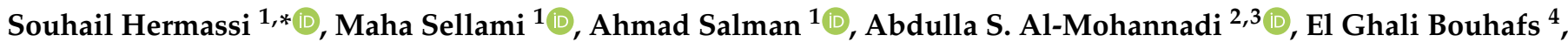 \\ Lawrence D. Hayes ${ }^{5}$ (D) and René Schwesig ${ }^{6}$ (D)
}

Citation: Hermassi, S.; Sellami, M.; Salman, A.; Al-Mohannadi, A.S.; Bouhafs, E.G.; Hayes, L.D.; Schwesig, R. Effects of COVID-19 Lockdown on Physical Activity, Sedentary Behavior, and Satisfaction with Life in Qatar: A Preliminary Study. Int. J. Environ. Res. Public Health 2021, 18, 3093. https:// doi.org/10.3390/ijerph18063093

Academic Editors: Elena

N. Naumova and Meghan

A. Hartwick

Received: 11 January 2021

Accepted: 17 February 2021

Published: 17 March 2021

Publisher's Note: MDPI stays neutra with regard to jurisdictional claims in published maps and institutional affiliations.

Copyright: (c) 2021 by the authors Licensee MDPI, Basel, Switzerland. This article is an open access article distributed under the terms and conditions of the Creative Commons Attribution (CC BY) license (https:// creativecommons.org/licenses/by/ $4.0 /)$
1 Physical Education Department, College of Education, Qatar University, Doha 2713, Qatar; msellami@qu.edu.qa (M.S.); asalman@qu.edu.qa (A.S.)

2 World Innovation Summit for Health (WISH), Qatar Foundation, Doha 5825, Qatar; absalmohannadi@qf.org.qa

3 Aspetar Orthopaedic and Sports Medicine Hospital, Doha 29222, Qatar

4 Department of Sports Science, Martin-Luther-University Halle-Wittenberg, 06120 Halle, Germany; bouhafs.elghali@gmail.com

5 School of Health and Life Sciences, University of the West of Scotland, Glasgow G72 0LH, UK. Lawrence.Hayes@uws.ac.uk

6 Department of Orthopaedic and Trauma Surgery, Martin-Luther-University Halle-Wittenberg, 06120 Halle, Germany; rene.schwesig@uk-halle.de

* Correspondence: shermassi@qu.edu.qa

Abstract: This study examined the effects of home confinement on physical activity (PA) and life satisfaction during the COVID-19 outbreak in Qatar. A total of 1144 subjects participated (male: $\mathrm{n}=588$; female: $\mathrm{n}=556$; age: $33.1 \pm 11.1$ years; mass: $76.1 \pm 16.4 \mathrm{~kg}$; height: $1.70 \pm 0.11 \mathrm{~m}$; body mass index (BMI): $26.1 \pm 4.44 \mathrm{~kg} / \mathrm{m}^{2}$ ). Online survey questions considered "before" and "during" confinement. Confinement reduced all PA intensities $\left(\eta_{\mathrm{p}}{ }^{2}=0.27-0.67, p<0.001\right)$ and increased daily sitting time from $3.57 \pm 1.47$ to $6.32 \pm 1.33$ h per weekday $\left(\eta_{p}{ }^{2}=0.67, p<0.001\right)$. The largest reduction was detected for the sum parameter all physical activity (minutes per week, $\eta_{\mathrm{p}}{ }^{2}=0.67, p<0.001$; MET (metabolic equivalent of task)-minutes / week, $\eta_{\mathrm{p}}{ }^{2}=0.69, p<0.001$ ). Life satisfaction decreased, with the score for "I am satisfied with my life" $\left(\eta_{\mathrm{p}}{ }^{2}=0.76, p<0.001\right)$ decreasing from $28.1 \pm 4.81$ to $14.2 \pm 6.41$ arbitrary units (AU). Concerning life satisfaction, the largest change was detected for the statement "the conditions of my life are excellent" $\left(d_{\text {male }}=7.93\right)$. For all parameters, time effects were indicative of large negative effects in both genders. In terms of magnitude, the difference between gender was greatest for the parameter "the conditions of my life are excellent" (difference between groups, $\mathrm{d}=4.84$ ). In conclusion, COVID-19 confinement decreased PA, increased sitting time, and decreased life satisfaction in Qatar. These precautionary findings explicate the risk of psychosocial impairment and the potential physical harm of reducing physical activity during early COVID-19 confinement in 2020 .

Keywords: SARS-CoV-2; lockdown; public health; physical activity; sedentary behavior; home confinement; lifestyle and contentment

\section{Introduction}

The World Health Organization (WHO) declared a pandemic due to COVID-19 on the 11 March 2020. Subsequently, the WHO recommended adopting several protective, behavioral, and non-pharmacological actions such as avoidance of physical contact, handshakes, closing universities and schools, banning social and large gatherings, and implementing self-isolation, social/physical distancing, confinement, and quarantine.

In the face of the ongoing pandemic, public health authorities and governments have enforced increasingly restrictive recommendations, including self-isolation, quarantine and 
even lockdowns of entire communities and territories [1,2]. While these restrictions help curb infection rate, such limitations may negatively affect participation in daily activities, physical activity (PA), traveling, and access to many forms of unstructured/structured exercise (e.g., closed gyms, no group gatherings, increased social/physical distancing) [1,2]. Numerous communities implemented curfews, limiting time for outdoor activities, or banned outdoor activities entirely. Such restrictions inflict a significant burden on population health by possibly compromising physical fitness, which is known to positively influence the ability to fight infections and reduce more severe immunologic and cardiopulmonary complications [3-5].

Regular PA induces cardioprotective physiological adaptations such as increased stroke volume, cardiac output, lowered resting heart rate and blood pressure, which improve individuals' health-related quality of life [6]. Prolonged homestay could increase behaviors that lead to physical inactivity and increase stress, which in turn, could increase the risk of several chronic health conditions that adversely affect health-related quality of life [6]. For example, limiting daily activity has been found to significantly impair health-related quality of life among severe acute respiratory syndrome (SARs) patients in Hong Kong [7].

The mandated restrictions concerning engagement in outdoor activities, including regular practice of exercise and physical activity in the time of the COVID-19 outbreak, are reducing exercising and increasing sedentary behavior, which can consequently contribute to anxiety, depression and common chronic health diseases [2,8-10]. In addition, restrictions likely reduced PA frequency and duration among typically active people who were unable to access gyms and health clubs and among those who achieve sufficient levels of PA incidentally, through walking or cycling to work or study [11,12]. It has been previously reported that COVID-19 confinement produced negative psychological effects, including post-traumatic stress, confusion, or anger [13,14]. During confinement, most individuals are living in an unprecedented situation of unknown duration, being exposed to anxiety, fear, depression, or sleep disruption [15,16].

It is important to note that both governmental policies and public behaviors are likely to vary and change over time depending on the situation of the COVID-19 pandemic. Based on preliminary responses ( 1000 participants), the present manuscript aimed to provide insight into the effect of home confinement on PA, sedentary behavior, and life satisfaction. Results from this study may provide guidance related to PA promotion and quality of life improvements, with the ultimate goal of establishing support programs for individuals living during the pandemic. We hypothesized that COVID-19 confinement would, independent of gender, (a) negatively impact PA participation, (b) negatively impact sedentary behavior, and (c) negatively impact life satisfaction. Additionally, we expect this to negatively impact of general population of Qatar state, especially amongst those who reduced their PA levels and increased their sedentary behavior.

\section{Materials and Methods}

An important criterion for selecting questionnaires was the proof and evidence of validity and reliability $[17,18]$. In this cross-sectional study using a convenience sample, we communicate preliminary findings of the first 1144 responses to an international online survey based on the International Physical Activity Questionnaire Short Form (IPAQ-SF) and Satisfaction with Life Questionnaire (SLQ), which were opened on 22 July 2020, tested by the project's steering group for one week and spread on 29 July 2020. During data collection in July 2020, the government of Qatar entered its second stage of the normalization plan and adopted a flexible lockdown. The IPAQ and SLQ were administered in English and Arabic. The survey included 22 questions on gender, demographic information (e.g., age, body mass and height), athlete (e.g., defined as a person who competes in one or more activities that involves physical strength, speed, and/or endurance), smoking status, health status (e.g., anxiety or depression, diabetes, cardiovascular disease and pulmonary disease, motor problem), PA (e.g., vigorous, moderate and walking activity) and SLQ (e.g., 
life conditions, satisfaction with life, and important things for life). Questions were to be answered directly in sequence regarding "before" and "during" confinement conditions [2] All measures were collected on the same day to avoid order or recall bias in the study, considering the continually evolving situation of the pandemic. Once the deadline for admitting surveys had passed, contradictory responses (incongruence between data), and repeated responses ( $\geq 2$ submissions with identical responses in a short period), were removed from the database. The study was completed in accordance with the Declaration of Helsinki. The present study's protocol was approved by the university's institutional review board (QU-IRB 1350EA-2020).

\subsection{Sample Size}

The sample size was calculated according to the following predictive equation [19].

$$
\mathrm{N}=((\mathrm{Z} \alpha / 22 \mathrm{p} \mathrm{q})) / \Delta 2
$$

$\mathrm{N}$ : number of needed participants,

$\mathrm{Z} \alpha / 2$ : two-tailed normal deviate for type 1 error,

p: change in \% from "before" to "during" confinement,

q: equal to " $1-\mathrm{p}$ " and $\Delta$ : accuracy; where " $\mathrm{n}$ " was the number of needed participants,

" $\mathrm{Z} \alpha / 2$ " was the two-tailed normal deviate for type 1 error $(\mathrm{Z} \alpha / 2=1.96$ for $95 \%$ level of significance),

" $q$ " was equal to " $1-\mathrm{p}$ ",

" $\Delta$ " was the accuracy $(=3 \%)$, and

" $p$ " was the percentage of change in social participation from "before" to "during" confinement period.

Comparable to Ammar et al. [2], the "p" was chosen from a study [20]. Zhang and Ma [20] examined the immediate effects of the COVID-19 pandemic on mental health and quality of life. Based on these findings, it appeared that $57.8 \%(p=0.578)$ of subjects experienced an increase in shared feelings with family members [20]. Consequently, the calculated sample size was $n=1041$. Assuming a dropout rate of $20 \%(n=208)$, we aimed to recruit 1250 participants.

\subsection{Survey Development, Promotion, and Distribution}

A steering group of academics and scientists (human science, sport science, and computer science) conceived the electronic survey at the University of Qatar (principal investigator). The survey was subsequently evaluated and amended by $>30$ colleagues and experts. The survey was uploaded and shared via the Google ${ }^{\mathrm{TM}}$ platform. The consortium distributed the electronic survey uniform resource locator (URL) via various methods: e-mails, shared on the consortium's faculties official pages, ResearchGate ${ }^{\mathrm{TM}}$, LinkedIn ${ }^{\mathrm{TM}}$, and other social media platforms such as Facebook ${ }^{\mathrm{TM}}$, Twitter ${ }^{\mathrm{TM}}$, and WhatsApp ${ }^{\mathrm{TM}}$.

The general public assisted in dissemination through the promotion of the survey within their networks. In total, the URL of the online survey was sent to 1250 potential participants, of which 1144 returned completed questionnaires and were included in the analysis (participation rate of $92 \%$ ). An introductory page described the survey background and aims, the consortium, ethics information, and the option to choose one of two available languages (English and Arabic). Inclusion criterion was that participants were aged $\geq 18$ years and in good health (no pain and diagnosis at the time of examination). Exclusion criteria included a positive COVID-19 test or existence of cognitive decline.

\subsection{Data Privacy/Security}

Participants were assured all data would be used exclusively for research purposes. Participants' responses were anonymous and confidential according to Google's privacy policy (https:/ / policies.google.com/privacy?hl=en, accessed on 22 July 2020). Participants were unable to provide names or contact information to ensure anonymity. Participants were free to withdraw and leave the questionnaire at any time before submission of 
responses. If they did so, responses would not be saved. Responses were saved once the "submit" button was pressed. By completing the survey, participants acknowledged the approval form and consented to voluntarily participate in this anonymous study. Participants were instructed to be honest with their responses.

\subsection{International Physical Activity Questionnaire Short Form (IPAQ-SF)}

According to the official IPAQ-SF recommendations, data are summed within each item (i.e., vigorous intensity, moderate intensity, and walking) to estimate the total amount of time engaged in PA per week $[17,18]$. Weekly PA (MET-min.week ${ }^{-1}$ ) was calculated by summing products of each PA item by a MET value specific to each category of PA. We assigned two sets of MET values. The first was the original values based on official IPAQ procedures for young and middle-aged adults (18-65 years old): vigorous PA = 8.0 METs, moderate PA = 4.0 METs and walking = 3.3 METs. Additionally, we added the total PA (sum of performed vigorous, moderate and walking activity) as a fourth item and sitting time as the fifth item of sedentary behavior assessed using the question: "Since self-isolating, how much time have you spent sitting daily?"

\subsection{Satisfaction with Life Questionnaire (SLQ)}

The Satisfaction of Life Questionnaire (SLQ) is a crisis-oriented questionnaire to assess satisfaction with the respondent's life before and during the confinement period. The SLQ is based on a 5-item scale designed to measure global cognitive judgments of one's life satisfaction. Participants indicate how much they agree or disagree with each of the 5 items using a 7-point scale that ranges from 7 strongly agree to 1 strongly disagree. Though scoring should be kept continuous (sum up scores on each item), here are some cut-offs to be used as benchmarks [21]. Using the 1-7 scale below, participants indicated their agreement with each of the five items (Strongly agree $=7$; Agree $=6$; Slightly agree $=5$; Neither agree nor disagree $=4$; Slightly disagree $=3$; Disagree $=2$; Strongly disagree $=1$ ) The total score of this questionnaire is comprised of the sum of scores from individual items. The total score for the SLQ ranges from 5 to 35, with lower scores corresponding to extreme dissatisfaction and higher scores with extreme satisfaction.

\subsection{Statistical Analyses}

All statistical analyses were performed using SPSS version 25.0 for Windows (SPSS Inc., IBM, Armonk, NY, USA). Means and standard deviations of dependent variables were calculated across participants. The chi square test was used to investigate gender differences for dichotomously or ordinally scaled data. Before analyses, all variables were tested for normal distribution (Shapiro-Wilk Test). Differences between males and females and between sessions (before vs. during confinement) were tested using a two-factor (gender, time), univariate general linear model. Within this analysis of variance (ANOVA) $p$-values and partial eta-squared $\left(\eta_{\mathrm{p}}{ }^{2}\right)$ were calculated using the Greenhouse-Geisser Test [22]. In this context, we reported group (gender), time (before, during confinement) and interaction (gender $x$ time) effects.

The effect size (the mean difference between scores divided by the pooled standard deviation) was also calculated for each parameter [23]. A positive effect size means an improvement of performance and a negative value indicates a decrease in performance. Percentage changes were calculated as ((before value - during value)/before value) $\times 100$. The interpretation of effect sizes is based on Cohen's thresholds for small effects $(\mathrm{d}<0.5)$, moderate effects $(d \geq 0.5)$ and large effects $(d>0.8)$ [24].

Differences between means (time effect) were considered as being meaningful if $p<0.001, \eta_{\mathrm{p}}{ }^{2}>0.10$ and the effect size (d) was $\geq 0.8$ [25]. Based on the number of parameters/tests and after applying a Bonferroni correction, we adjusted the $\alpha$ error level for both types of parameters (PA: $0.05 / 13=0.004$; satisfaction of life: $0.05 / 6=0.008$ ). In the interest of a uniform approach and conservative assessment of the effects, we defined an alpha error level of $p<0.001$. 


\section{Results}

\subsection{Sample Description}

1144 (female: $\mathrm{n}=588$; male: $\mathrm{n}=556$ ) participants were recruited in Qatar (Table 1).

Table 1. Demographic and anthropometric characteristics of all participants $(\mathrm{n}=1144)$. Significant effects (criteria of relevance: $p<0.05$ and $\eta_{p}{ }^{2}>0.10$ and $d>0.8$ ) highlighted in bold.

\begin{tabular}{cccccc}
\hline & $\begin{array}{c}\text { Total } \\
(\mathbf{n}=\mathbf{1 1 4 4})\end{array}$ & $\begin{array}{c}\text { Male } \\
(\mathbf{n}=5 \mathbf{5 8})\end{array}$ & $\begin{array}{c}\text { Female } \\
(\mathbf{n}=\mathbf{5 5 6})\end{array}$ & $\boldsymbol{p}$ & $\boldsymbol{\eta}_{\mathbf{p}}{ }^{2}$ \\
\hline \multirow{2}{*}{ Age $(\mathrm{yr})$} & $33.1 \pm 11.1$ & $\begin{array}{c}35.9 \pm 11.4 \\
(18.0-67.0)\end{array}$ & $\begin{array}{c}30.0 \pm 9.90 \\
(18.0-67.0)\end{array}$ & $<0.001$ & 0.07 \\
\hline \multirow{2}{*}{ Height $(\mathrm{m})$} & $\begin{array}{c}1.70 \pm 0.10 \\
(1.40-1.98)\end{array}$ & $\begin{array}{c}1.78 \pm 0.10 \\
(1.55-1.98)\end{array}$ & $\begin{array}{c}1.62 \pm 0.10 \\
(1.40-1.81)\end{array}$ & $<0.001$ & 0.53 \\
\hline \multirow{2}{*}{ Mass $(\mathrm{kg})$} & $\begin{array}{c}76.1 \pm 16.4 \\
(28.0-142)\end{array}$ & $\begin{array}{c}85.4 \pm 13.1 \\
(50.0-142)\end{array}$ & $\begin{array}{c}66.2 \pm 13.4 \\
(28.0-140)\end{array}$ & $<0.001$ & \multirow{2}{*}{0.34} \\
\hline \multirow{2}{*}{ BMI $\left(\mathrm{kg} / \mathrm{m}^{2}\right)$} & $\begin{array}{c}26.1 \pm 4.44 \\
(11.7-49.1)\end{array}$ & $\begin{array}{c}27.0 \pm 4.15 \\
(16.4-49.1)\end{array}$ & $\begin{array}{c}25.1 \pm 4.54 \\
(11.7-44.7)\end{array}$ & $<0.001$ & \multirow{2}{*}{0.04} \\
\hline
\end{tabular}

Results reported as mean \pm standard deviation (range). BMI = body mass index.

Significant differences were observed between males and females for height $(p<0.001$, $\left.\eta_{\mathrm{p}}{ }^{2}=0.53\right)$ and body mass $\left(p<0.001, \eta_{\mathrm{p}}{ }^{2}=0.34\right)$ but not for age $\left(p<0.001, \eta_{\mathrm{p}}{ }^{2}=0.07\right)$. BMI was the anthropometric parameter with the smallest difference $\left(p<0.001, \eta_{p}{ }^{2}=0.04\right)$ between males and females.

We found a gender effect (Chi-Square $=111 ; p<0.001)$, with the proportion of athletes amongst women higher $(45 \% ; 250 / 556)$ than among men $(16 \% ; 96 / 588)$. An effect of gender was observed for number of smokers (Chi-Square $=334.2 ; p<0.001)$. The percentage of smokers was different between women $(0 \%)$ and men $(46 \% ; 270 / 588)$. Most subjects ( $76 \%$; $878 / 1144$ ) reported no health problems (Table 2). Anxiety or depression was the most prevalent health problem reported among the sample $(14 \% ; 158 / 1144)$.

Table 2. Description of the sample ( $n=1144$; female: $n=556$, male: $n=588$ ) regarding demography, anthropometry and health status depending on gender.

\begin{tabular}{|c|c|c|c|c|c|}
\hline Variables & Categories & Male n (\%) & Female n (\%) & Total $\mathbf{n}$ & $\mathrm{Chi}^{2}(p)$ \\
\hline \multirow{3}{*}{ Age (yr) } & $18-35$ & $308(43)$ & 409 (57) & 717 & \multirow{3}{*}{$55.7<0.001$} \\
\hline & $36-55$ & $255(65)$ & $138(35)$ & 393 & \\
\hline & $>55$ & $25(74)$ & $9(26)$ & 34 & \\
\hline \multirow{4}{*}{ BMI $\left(\mathrm{kg} / \mathrm{m}^{2}\right)$} & $<18.5$ & $5(33)$ & $10(67)$ & 15 & \multirow{4}{*}{$43.6<0.001$} \\
\hline & $18.5-24.9$ & $206(41)$ & 297 (59) & 503 & \\
\hline & $25-29.9$ & $259(60)$ & $175(40)$ & 434 & \\
\hline & 30 or greater & $118(62)$ & $74(38)$ & 192 & \\
\hline \multirow{2}{*}{ Athlete } & Yes & $96(28)$ & $250(72)$ & 346 & \multirow{2}{*}{$111.1<0.001$} \\
\hline & No & $492(62)$ & $306(38)$ & 798 & \\
\hline \multirow{2}{*}{ Smoker } & Yes & $270(100)$ & $0(0)$ & 270 & \multirow{2}{*}{$334.2<0.001$} \\
\hline & No & $318(36)$ & $556(64)$ & 874 & \\
\hline \multirow{6}{*}{ Health status } & None of the above & $452(52)$ & $426(68)$ & 878 & \multirow{6}{*}{$93.8<0.001$} \\
\hline & $\begin{array}{l}\text { Anxiety or } \\
\text { depression }\end{array}$ & $117(74)$ & $41(26)$ & 158 & \\
\hline & Diabetes & $0(0)$ & $19(100)$ & 19 & \\
\hline & Cardiovascular & & & & \\
\hline & $\begin{array}{c}\text { disease and } \\
\text { pulmonary disease }\end{array}$ & $0(0)$ & $32(100)$ & 32 & \\
\hline & Motor problem & $19(33)$ & $38(67)$ & 57 & \\
\hline
\end{tabular}


3.2. International Physical Activity Questionnaire Short Form (IPAQ-SF)_-Testing of Hypotheses 1 and 2

The time effects for the PA parameters (vigorous or moderate physical activities and walking) and sedentary behavior (measured by sitting time) ranged from $\eta_{\mathrm{p}}{ }^{2}=0.27$ (vigorous minutes per week) to $\eta_{p}{ }^{2}=0.67$ (sitting; hours per weekday; Table 3 ). The paired effect sizes (d) considering before vs. during confinement were similar in both sexes and ranged from $d=0.58$ (female, vigorous physical activities $/$ minutes/week) to $d=2.17$ (male, sitting/hours per weekday). The greatest difference between male $(\mathrm{d}=1.97)$ and female $(\mathrm{d}=1.30)$ was observed in moderate PA (MET-minutes/week). Significant gender effects (Table 3$)$ were observed for $46 \%(6 / 13)$ of the investigated parameters. No interaction effects (gender $x$ time) were observed.

Table 3. Comparison of physical activity parameters and sedentary behavior (sitting time) between males and females before and during confinement. Values are given as mean \pm SD. Significant effects (criteria of relevance: $p<0.05$ and $\eta_{\mathrm{p}}{ }^{2}>$ 0.10 and $d>0.8$ ) highlighted in bold.

\begin{tabular}{|c|c|c|c|c|c|c|c|c|c|}
\hline & \multicolumn{3}{|c|}{ Male $(n=588)$} & \multicolumn{3}{|c|}{ Female $(n=556)$} & \multicolumn{3}{|c|}{ Variance Analysis/Effects $p\left(\eta_{\mathrm{p}}{ }^{2}\right)$} \\
\hline & Before & During & d & Before & During & d & Gender & Time & $\begin{array}{l}\text { Gender } \\
\times \text { Time }\end{array}$ \\
\hline \multicolumn{10}{|c|}{ Vigorous physical activities } \\
\hline Days/week (d) & $\begin{array}{c}2.09 \pm \\
0.81\end{array}$ & $\begin{array}{c}1.43 \pm \\
0.56\end{array}$ & 0.96 & $\begin{array}{c}2.44 \pm \\
1.00\end{array}$ & $\begin{array}{c}1.46 \pm \\
0.82\end{array}$ & 1.08 & $\begin{array}{l}<0.001 \\
(0.03)\end{array}$ & $\begin{array}{l}<0.001 \\
(0.35)\end{array}$ & $\begin{array}{l}<0.001 \\
(0.02)\end{array}$ \\
\hline $\begin{array}{l}\text { Minutes/week } \\
\text { (min) }\end{array}$ & $\begin{array}{c}39.7 \pm \\
11.3\end{array}$ & $\begin{array}{c}28.0 \pm \\
10.5\end{array}$ & 1.07 & $\begin{array}{c}25.7 \pm \\
12.1\end{array}$ & $\begin{array}{c}19.7 \pm \\
8.48\end{array}$ & 0.58 & $\begin{array}{l}<0.001 \\
(0.34)\end{array}$ & $\begin{array}{l}<0.001 \\
(0.27)\end{array}$ & $\begin{array}{l}<0.001 \\
(0.04)\end{array}$ \\
\hline $\begin{array}{c}\text { MET- } \\
\text { minutes/week }\end{array}$ & $663 \pm 320$ & $323 \pm 187$ & 1.34 & $520 \pm 372$ & $238 \pm 205$ & 0.98 & $\begin{array}{l}<0.001 \\
(0.07)\end{array}$ & $\begin{array}{c}<0.001 \\
(0.39)\end{array}$ & $\begin{array}{l}0.012 \\
(0.01)\end{array}$ \\
\hline \multicolumn{10}{|c|}{ Moderate physical activities } \\
\hline Days/week (d) & $\begin{array}{c}2.42 \pm \\
0.66\end{array}$ & $\begin{array}{c}1.56 \pm \\
0.54\end{array}$ & 0.99 & $\begin{array}{c}2.16 \pm \\
0.82\end{array}$ & $\begin{array}{c}1.46 \pm \\
0.63\end{array}$ & 0.97 & $\begin{array}{l}<0.001 \\
(0.03)\end{array}$ & $\begin{array}{l}<0.001 \\
(0.41)\end{array}$ & $\begin{array}{l}0.003 \\
(0.01)\end{array}$ \\
\hline $\begin{array}{l}\text { Minutes/week } \\
\text { (min) }\end{array}$ & $\begin{array}{c}40.4 \pm \\
11.2\end{array}$ & $\begin{array}{c}24.2 \pm \\
9.05\end{array}$ & 1.60 & $\begin{array}{c}35.3 \pm \\
14.3\end{array}$ & $\begin{array}{c}21.0 \pm \\
9.51\end{array}$ & 1.20 & $\begin{array}{l}<0.001 \\
(0.06)\end{array}$ & $\begin{array}{l}<0.001 \\
(0.50)\end{array}$ & $\begin{array}{l}0.035 \\
(0.00)\end{array}$ \\
\hline $\begin{array}{c}\text { MET- } \\
\text { minutes/week }\end{array}$ & $394 \pm 166$ & $152 \pm 79.7$ & 1.97 & $315 \pm 188$ & $130 \pm 96.3$ & 1.30 & $\begin{array}{l}<0.001 \\
(0.06)\end{array}$ & $\begin{array}{l}<0.001 \\
(0.54)\end{array}$ & $\begin{array}{l}<0.001 \\
(0.02)\end{array}$ \\
\hline \multicolumn{10}{|c|}{ Walking } \\
\hline $\begin{array}{l}\text { Days / walk for } \\
\text { at least } 10 \mathrm{~min} \\
\text { (d) }\end{array}$ & $\begin{array}{c}4.28 \pm \\
1.47\end{array}$ & $\begin{array}{c}2.74 \pm \\
0.74\end{array}$ & 1.39 & $\begin{array}{c}3.06 \pm \\
1.09\end{array}$ & $\begin{array}{c}2.07 \pm \\
0.92\end{array}$ & 0.99 & $\begin{array}{l}<0.001 \\
(0.24)\end{array}$ & $\begin{array}{l}<0.001 \\
(0.45)\end{array}$ & $\begin{array}{l}<0.001 \\
(0.04)\end{array}$ \\
\hline $\begin{array}{l}\text { Minutes } \\
\text { per/walking } \\
\text { days (min) }\end{array}$ & $\begin{array}{c}37.0 \pm \\
12.5\end{array}$ & $\begin{array}{c}23.0 \pm \\
8.59\end{array}$ & 1.33 & $\begin{array}{c}43.5 \pm \\
17.2\end{array}$ & $\begin{array}{c}20.6 \pm \\
8.73\end{array}$ & 1.77 & $\begin{array}{l}<0.001 \\
(0.01)\end{array}$ & $\begin{array}{l}<0.001 \\
(0.57)\end{array}$ & $\begin{array}{l}<0.001 \\
(0.07)\end{array}$ \\
\hline $\begin{array}{c}\text { MET- } \\
\text { minutes/week }\end{array}$ & $528 \pm 271$ & $215 \pm 120$ & 1.60 & $449 \pm 261$ & $141 \pm 87.0$ & 1.77 & $\begin{array}{l}<0.001 \\
(0.06)\end{array}$ & $\begin{array}{c}<0.001 \\
(0.58)\end{array}$ & $\begin{array}{l}0.767 \\
(0.00)\end{array}$ \\
\hline \multicolumn{10}{|c|}{ Sitting } \\
\hline $\begin{array}{c}\text { Hours per } \\
\text { weekday (h) }\end{array}$ & $\begin{array}{c}3.64 \pm \\
1.42\end{array}$ & $\begin{array}{c}6.51 \pm \\
1.22\end{array}$ & 2.17 & $\begin{array}{c}3.49 \pm \\
1.53\end{array}$ & $\begin{array}{c}6.12 \pm \\
1.41\end{array}$ & 1.79 & $\begin{array}{c}<0.001 \\
(0.02)\end{array}$ & $\begin{array}{c}<0.001 \\
(0.67)\end{array}$ & $\begin{array}{l}0.040 \\
(0.00)\end{array}$ \\
\hline \multicolumn{10}{|c|}{ All Physical Activity } \\
\hline Days/week (d) & $\begin{array}{c}2.93 \pm \\
0.57\end{array}$ & $\begin{array}{c}1.91 \pm \\
0.37\end{array}$ & 2.17 & $\begin{array}{c}2.55 \pm \\
0.61\end{array}$ & $\begin{array}{c}1.67 \pm \\
0.53\end{array}$ & 1.54 & $\begin{array}{l}<0.001 \\
(0.13)\end{array}$ & $\begin{array}{l}<0.001 \\
(0.66)\end{array}$ & $\begin{array}{l}0.001 \\
(0.01)\end{array}$ \\
\hline $\begin{array}{c}\text { Minutes/week } \\
\text { (min) }\end{array}$ & $117 \pm 21.4$ & $\begin{array}{c}75.1 \pm \\
16.9\end{array}$ & 2.19 & $105 \pm 31.3$ & $\begin{array}{c}61.2 \pm \\
16.0\end{array}$ & 1.85 & $\begin{array}{l}<0.001 \\
(0.14)\end{array}$ & $\begin{array}{l}<0.001 \\
(0.67)\end{array}$ & $\begin{array}{l}0.468 \\
(0.00)\end{array}$ \\
\hline $\begin{array}{c}\text { MET- } \\
\text { minutes/week }\end{array}$ & $\begin{array}{c}1584 \pm \\
447\end{array}$ & $689 \pm 249$ & 2.57 & $\begin{array}{c}1283 \pm \\
582\end{array}$ & $509 \pm 271$ & 1.82 & $\begin{array}{l}<0.001 \\
(0.14)\end{array}$ & $\begin{array}{l}<0.001 \\
(0.69)\end{array}$ & $\begin{array}{l}<0.001 \\
(0.01)\end{array}$ \\
\hline
\end{tabular}




\subsection{Satisfaction with Life Questionnaire (SLQ)—Testing of Hypothesis 3}

The comparison of "satisfaction with life" parameters (Table 4) revealed an interaction (time $\times$ gender) effect $\left(\eta_{\mathrm{p}}{ }^{2}=0.14\right)$ for the statement "So far I have gotten the important things I want in life". The reduction was smaller in females (absolute difference $=13.4$, $\mathrm{d}=3.89$ ) than in males (absolute difference $=17.5, \mathrm{~d}=5.15$ ).

Table 4. Comparison of "satisfaction with life" parameters depending on sex before and during confinement. Values are given as mean \pm SD. Significant effects (criteria of relevance: $p<0.05$ and $\eta_{p}{ }^{2}>0.10$ and $d>0.8$ ) highlighted in bold.

\begin{tabular}{|c|c|c|c|c|c|c|c|c|c|}
\hline & \multicolumn{3}{|c|}{ Male (n = 588) } & \multicolumn{3}{|c|}{ Female $(n=556)$} & \multicolumn{3}{|c|}{ Variance Analysis/Effects $p\left(\eta_{p}{ }^{2}\right)$} \\
\hline & Before & During & d & Before & During & d & Sex & Time & $\begin{array}{c}\text { Sex } \times \\
\text { Time }\end{array}$ \\
\hline \multicolumn{10}{|c|}{ In most ways my life is close to my ideal } \\
\hline Score Q1 & $\begin{array}{c}29.8 \pm \\
5.06\end{array}$ & $\begin{array}{c}12.6 \pm \\
3.34\end{array}$ & 4.10 & $\begin{array}{c}29.5 \pm \\
4.85\end{array}$ & $\begin{array}{c}12.4 \pm \\
6.11\end{array}$ & 3.12 & $\begin{array}{l}0.230 \\
(0.00)\end{array}$ & $\begin{array}{c}<0.001 \\
(0.86)\end{array}$ & $\begin{array}{l}0.989 \\
(0.00)\end{array}$ \\
\hline \multicolumn{10}{|c|}{ The conditions of my life are excellent. } \\
\hline Score Q2 & $\begin{array}{c}33.1 \pm \\
2.63\end{array}$ & $\begin{array}{c}12.2 \pm \\
2.64\end{array}$ & 7.93 & $\begin{array}{c}30.3 \pm \\
4.15\end{array}$ & $\begin{array}{c}13.2 \pm \\
6.92\end{array}$ & 3.09 & $\begin{array}{c}<0.001 \\
(0.02)\end{array}$ & $\begin{array}{c}<0.001 \\
(0.91)\end{array}$ & $\begin{array}{l}<0.001 \\
(0.09)\end{array}$ \\
\hline \multicolumn{10}{|c|}{ I am satisfied with my life } \\
\hline Score Q3 & $\begin{array}{c}28.8 \pm \\
4.26\end{array}$ & $\begin{array}{c}16.4 \pm \\
5.25\end{array}$ & 2.61 & $\begin{array}{c}27.5 \pm \\
5.26\end{array}$ & $\begin{array}{c}11.8 \pm \\
6.67\end{array}$ & 2.63 & $\begin{array}{c}<0.001 \\
(0.14)\end{array}$ & $\begin{array}{c}<0.001 \\
(0.76)\end{array}$ & $\begin{array}{l}<0.001 \\
(0.04)\end{array}$ \\
\hline \multicolumn{10}{|c|}{ So far I have gotten the important things I want in life. } \\
\hline Score Q4 & $\begin{array}{c}30.5 \pm \\
4.02\end{array}$ & $\begin{array}{c}13.0 \pm \\
2.78\end{array}$ & 5.15 & $\begin{array}{c}28.6 \pm \\
2.86\end{array}$ & $\begin{array}{c}15.2 \pm \\
4.03\end{array}$ & 3.89 & $\begin{array}{l}0.241 \\
(0.00) \\
\end{array}$ & $\begin{array}{c}<0.001 \\
(0.90)\end{array}$ & $\begin{array}{l}<0.001 \\
(0.14)\end{array}$ \\
\hline \multicolumn{10}{|c|}{ If I could live my life over, I would change almost nothing. } \\
\hline Score Q5 & $\begin{array}{c}31.8 \pm \\
3.56\end{array}$ & $\begin{array}{c}15.0 \pm \\
4.52\end{array}$ & 4.94 & $\begin{array}{c}31.1 \pm \\
4.73\end{array}$ & $\begin{array}{c}10.9 \pm \\
5.54\end{array}$ & 3.93 & $\begin{array}{c}<0.001 \\
(0.12)\end{array}$ & $\begin{array}{c}<0.001 \\
(0.89)\end{array}$ & $\begin{array}{l}<0.001 \\
(0.06)\end{array}$ \\
\hline \multicolumn{10}{|c|}{ Total score } \\
\hline & $\begin{array}{c}30.8 \pm \\
2.00\end{array}$ & $\begin{array}{c}13.8 \pm \\
1.77\end{array}$ & 9.02 & $\begin{array}{c}29.4 \pm \\
1.85\end{array}$ & $\begin{array}{c}12.7 \pm \\
2.54\end{array}$ & 7.61 & $\begin{array}{c}<0.001 \\
(0.17)\end{array}$ & $\begin{array}{c}<0.001 \\
(0.97)\end{array}$ & $\begin{array}{l}0.117 \\
(0.00)\end{array}$ \\
\hline
\end{tabular}

The time effects reached the $p<0.05$ level in all parameters and ranged from $\eta_{\mathrm{p}}{ }^{2}=0.76$ (I am satisfied with my life. Figure 1) to $\eta_{\mathrm{p}}{ }^{2}=0.91$ (The conditions of my life are excellent. Figure 2). The results concerning "In most ways my life is close to my ideal" (time effect: $\eta_{\mathrm{p}}{ }^{2}=0.86$; Figure 3 ) and "If I could live my life over, I would change almost nothing" (time effect: $\eta_{\mathrm{p}}{ }^{2}=0.89$ ) were similar. Regarding these two questions, effect size in the subgroups ranged from $\mathrm{d}=3.12$ to $\mathrm{d}=4.94$ (Table 4; Figure 3).

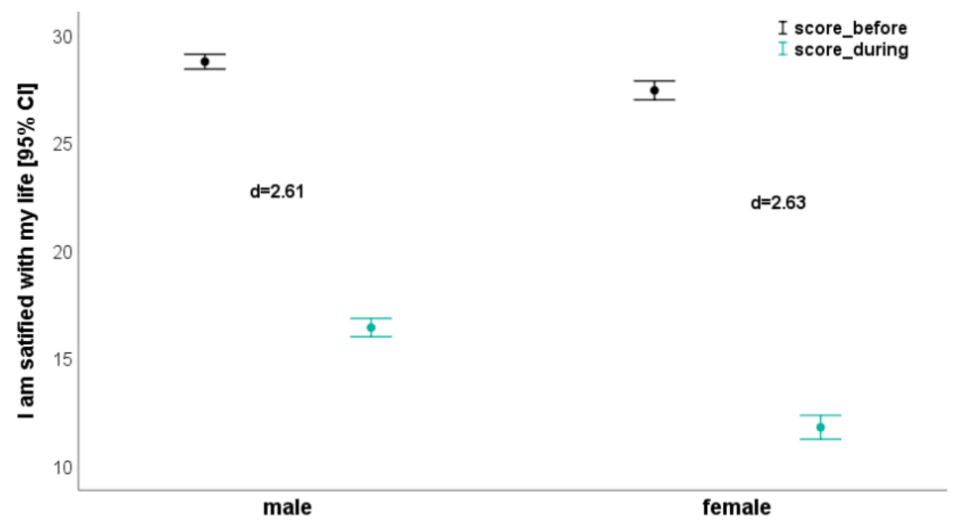

Figure 1. I am satisfied with my life-depending on gender before and during confinement. 


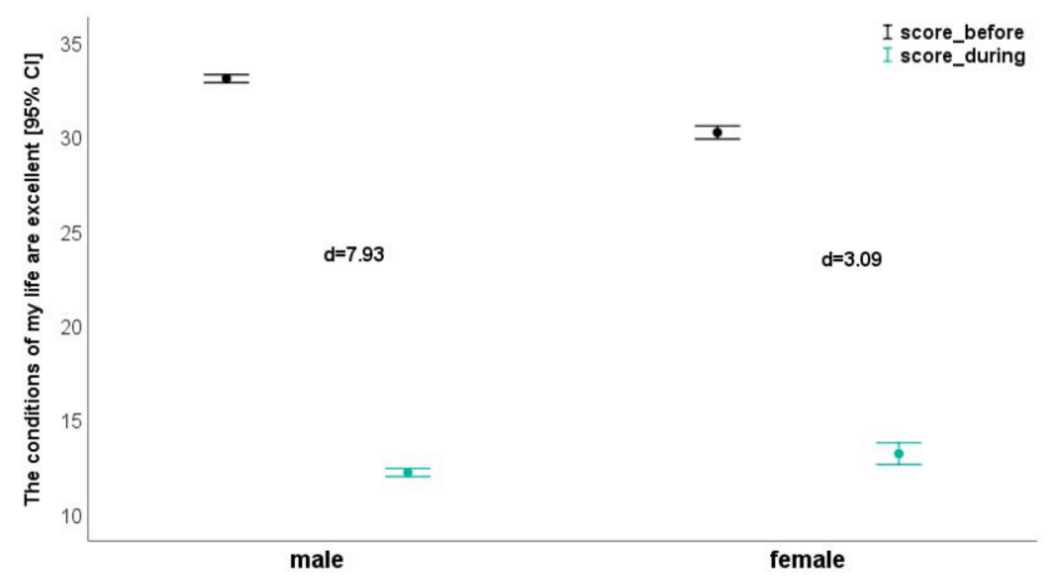

Figure 2. The conditions of my life are excellent—depending on gender before and during confinement.

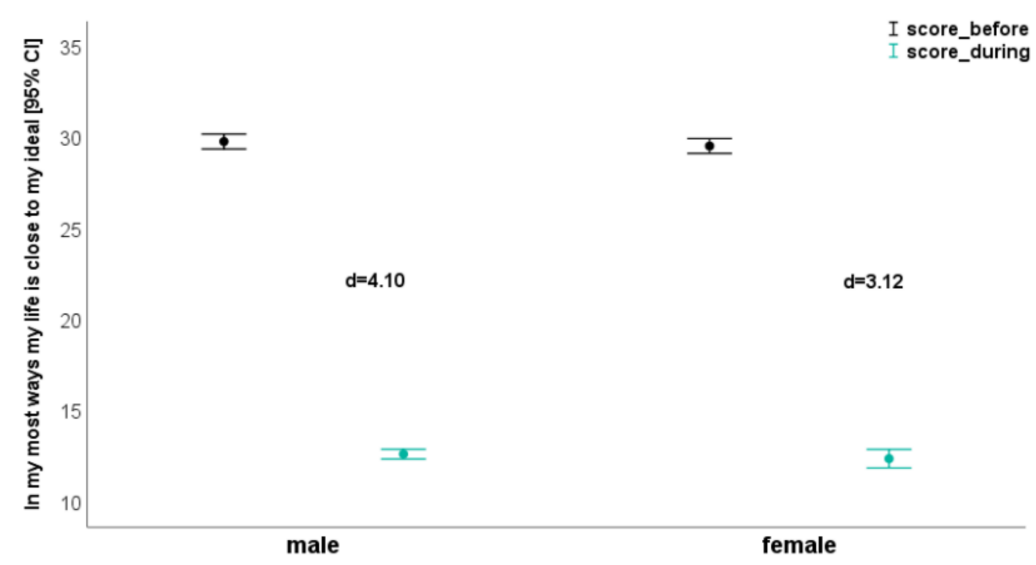

Figure 3. In my most ways, my life is close to my ideal-depending on gender before and during confinement).

The total satisfaction with life demonstrated the greatest reduction in both males $(\mathrm{d}=9.02$; from $30.8 \pm 2.00$ to $13.8 \pm 1.77)$ and females $(\mathrm{d}=7.61$; from $29.4 \pm 1.85$ to $12.7 \pm 2.54)$.

\section{Discussion}

To attenuate COVID-19 spread, policymakers have implemented restrictive measures in many countries. There are little data on the precise impact of this crisis on population mental health, whether in the short, medium or long term. If links between COVID-19 and mental and behavioral health symptoms are detected, they remain to be validated by large scale cross-sectional or longitudinal studies. During times of crisis, the implementation of severe restrictions and strategies to control and mitigate the spread of disease may have short term or long-term implications on mental health and physical activity. While these restrictions may be intended to protect the publics' physical health, it may be detrimental to their mental health and well-being. With this in mind, understanding the psychosocial implications of home isolation would provide data on the impact of such health measures on lifestyle (especially physical activity, physical and mental health).

The present study aimed to elucidate the effect of home confinement on PA and life satisfaction based on data extracted from the first thousand responses in Qatar. Home confinement by COVID-19 caused a significant decrease in walking per week (assumption of hypothesis 1) and an increase in sedentary behavior measured by sitting time (assumption of hypothesis 2), which reflects PA in daily life. In addition, PA at vigorous and moderate intensity was decreased by COVID-19 home confinement. Similarly, the total score for life satisfaction decreased by $\sim 56 \%$ (male: $55 \%$; female: $57 \%$ ). Due to the negative impact upon 
social participation and life satisfaction (assumption of hypothesis 3), data presented here support the conclusion that there is significant risk of psychosocial strain during home confinement conditions caused by the pandemic.

\subsection{Impact of COVID 19 on Physical Activity}

The positive health benefits of PA and sedentary behavior in daily life are well established and there is clear evidence linking physical inactivity to non-communicable diseases [26]. In addition, many governmental agencies have developed PA guidelines not only as a preventive strategy for chronic diseases, but also for psychological benefits [27-29]. Recent multicenter studies showed that COVID-19 home confinement increased the numbers of physically inactive individuals $(+15 \%)[2,11]$. In this study, the number of walking days for at least 10 min per week was decreased of $~ 35 \%$ in both genders. Concomitantly, time per walk decreased of $46 \%$ in both genders. Therefore, the energy expenditure of walking per week was decreased from $449 \pm 261$ MET-minutes/week to $141 \pm 87.0$ MET-minutes/week for women and from $528 \pm 271$ MET-minutes/week to $215 \pm 120$ MET-minutes/week in men. It is thought that walking PA is part of the habitual activities of daily life. Thus, the decreased walking volume in our subjects may reflect an inactive lifestyle. Ammar et al. [30] reported after an international online survey was launched in April 2020 that the number of days/week of walking decreased by $35 \%$ during home confinement $(t=15.80, p<0.001, d=0.68)$. Likewise, the same study reported that the number of minutes/day of walking decreased by $34 \%$ during home confinement $(t=9.34, p<0.001, d=0.39)$. Additionally, MET values of walking were $43 \%$ lower during home confinement $(t=9.03, p<0.001, d=0.36)$. It is important to mention here that the studies were all developed during the cool weather, which make comparison easier between populations from North Africa, Europe and Asia.

Results of PA at vigorous intensities were consistent with those of previous studies in Italian athletes [31]. The frequency of vigorous intensity PA during home confinement decreased from $40 \%$ to $23 \%$ in women and from $32 \%$ to $30 \%$ in men, respectively. Giustino et al. [31] reported energy expenditures in vigorous intensity were decreased from $520 \pm 372$ MET-minutes/week to $238 \pm 205$ MET-minutes/week in women and from $663 \pm 320$ MET-minutes/week to $323 \pm 187$ MET-minutes/week in men by home confinement. However, Ammar et al. [30] indicate that the number of days/week and minutes/day of vigorous intensity PA during confinement decreased $23 \%(t=7.75, p<0.001, d=0.37)$ and $33 \%(t=9.75 p<0.001, d=0.54)$, respectively. Moreover, vigorous intensity METs were $37 \%$ lower during home confinement $(t=6.68, p<0.001, d=0.32)$ compared to before. In addition, in a survey of PA during lockdown in the Canadian adult population [12], vigorous PA among highly active people (Moderate to Vigorous Physical Activity (MVPA) of $302 \pm 186$ min per week) did not decreased despite the lockdown. Their vigorous PA consisted of individual outdoor exercise such as walking, running and cycling. In contrast, amounts of vigorous and moderate intensity PA in this study decreased. The difference between these results could be the difference between individual PA for recreation and health promotion. However, Ammar et al. [30] found that moderate intensity PA, in days per week, decreased by $24 \%$ during home confinement $(t=7.89, p<0.001, d=0.40)$. Likewise, minutes/day of moderate intensity PA decreased 33\% during home confinement $(\mathrm{t}=7.85, p<0.001, \mathrm{~d}=0.34)$. As a result, moderate intensity METs were $35 \%$ lower during confinement $(\mathrm{t}=5.24, p<0.001, \mathrm{~d}=0.20)$.

People undertaking individual PA for recreation and health promotion may continue the same exercise during lockdown because they are outdoor activities such as walking, running, and cycling. Conversely, individuals involved in indoor sports obtained their MVPA almost exclusively through handball training in training facilities that closed during restrictions. Most athletes who usually complete PA in training facilities have been forced to train at home or in their own backyards $[2,12,31]$. Thus, some of the athletes in this study would no longer be able to gather in training facilities for training and matches and likely trained individually in their own homes and/or backyards. This may have 
profound implications for the athletic participants within this study as the inability to maintain intensity and volume of habitual training would result in reduced physical performance [32-34]. Moreover, the transition back into sport-specific practices would result in a high risk of injury [35].

Sitting time would be considered an indicator of how much time is spent in the home, and we report herein that sitting time on weekdays increased almost twofold in both genders. Previous studies have reported healthy populations increased time watching television, social networking using smart phones, and video gaming during COVID-19 home confinement $[30,36]$. Ammar et al. [30] also reported the number of hours/day sitting increased $29 \%$ during home confinement. Thus, the impact of home confinement by COVID-19 on physical inactivity in daily life in this study is commensurate with results from earlier investigations.

Statistics from the current sample showed a slightly high percentage of individuals with depression and anxiety problems (anxiety or depression: 14\%). Such results were expected, as the current pandemic is a time of uncertainty and concern for many people. This can affect individuals on a physical level, but also on a psychological level. Indeed, in such a context, many people will experience reactions of stress, anxiety and depression. Interestingly, in our study the proportion of smokers $(24 \%)$, people with diabetes $(2 \%)$, people with cardiovascular disease and pulmonary disease (3\%) and motor problems $(5 \%)$ was much lower compared to the general population. Through recent decades, Qatar has developed a healthcare strategy for prevention, cure, and care to increase awareness of health and fitness [37] which has led to lower rates of mortality when infected [38].

\subsection{Impact COVID 19 on Life Satisfaction}

COVID-19 induced social isolation negatively impacted mental health. In 1006 Italians under COVID-19 quarantine, prolonged isolation increased depression, unworthiness, alienation, and helplessness [39]. Similarly, adults ceasing work after one month of confinement in China (2020) reported worse health circumstances and mental distress. The present findings revealed a significant interaction effect for the statement "So far I have gotten the important things I want in life" as the reduction was less in females than males. The time effect reached $p<0.05$ in all parameters (I am satisfied with my life, the conditions of my life are excellent). Ammar et al. [2] showed that the total score for Life Satisfaction decreased by $16 \%$ during home confinement. This overall score is a factor of three questions (Q1-Q3), and the decrease from "before" to "during" confinement ranged from $-14 \%$ to $-18 \%$. These negative effects have also been reported in a recent COVID-19 series highlighting that people under quarantine conditions report more symptoms of psychological distress. Furthermore, some symptoms appeared to persist after quarantine has ceased [40]. In China, COVID-19's resultant social distancing reduced life satisfaction and increased distress [41]. The present findings corroborate these previous reports, substantiating the risk of psychosocial strain during home confinement periods.

Ammar et al. [42] indicated that COVID-19 home confinement had a negative effect on mental health, mood and feelings. In terms of prevalence, more individuals $(+13 \%)$ reported lower mental wellbeing during, compared to before, home confinement. Mood and feelings responses showed a $45 \%$ increase in depressive symptoms, with more people $(+10 \%)$ showing depressive symptoms during, compared to before, home confinement. The ECLB-COVID-19 survey revealed an increased psychosocial strain triggered by home confinement. To mitigate this risk of poor mental health and to foster an active and healthy confinement lifestyle (AHCL), a crisis-oriented interdisciplinary intervention is urgently needed.

Associations between mental health and quality of life have recently been reported [43] However, factors such as income, which in turn is relates to factors such as education and health literacy, mediate associations between mental health and quality of life in SARs patients [44,45]. Apart from the above influences, Qi et al. [46] reported stress and healthrelated quality of life was strongly negatively associated among Chinese adults during 
the COVID-19 pandemic. Psychological perturbations in SARs infected patients during the quarantine periods appear relatively ubiquitous [47]. Home confinement negatively influences mental wellbeing and emotional status, potentially due to physical inactivity, social isolation, and unemployment [2]. Importantly, sedentary behavior due to COVID19 negatively impacted people's quality of life in this study. Therefore, it is critically important to promote people's health and wellbeing by encouraging them to minimize sitting time and keep physically active to maximize satisfaction with life during the COVID19 pandemic. Further studies are needed to confirm the association between stress and health-related quality of life during or after the COVID-19 pandemic.

\subsection{Strengths, Limitations, and Perspective}

The strength of this study was the rapid data generation during the pandemic using an anonymous cross-disciplinary survey provided in two languages, widely distributed over several region in Qatar. However, given the preliminary nature of this study, moderating effects of demographics, cultures, and age have not been studied. Regarding the methodological issues, possible limitations could be (i) the cross-sectional design assessing "before" home confinement condition retrospectively, and (ii) the disuse of cookie-based or I.P.-based duplicate protection to exclude duplicates. Our consortium elected to avoid I.P. or cookie safety measures, as we know that during home confinement more than one family member may use the same computer (e.g., same I.P.). Given that home confinement was a sudden measure in most countries, we were obviously unable to develop and spread the survey "before" home confinement, to avoid recall bias. Additionally, different types of PA, divergent professions, presence of children in the home, loss of job, and loss of friends or relatives may be confounding factors on SLQ and perceived stress, which were not accounted for in the present study due to the need for rapid data gathering, and these could be considered in future studies. The compliance regarding the rigid rules of lockdown is also an important predictor.

\section{Conclusions}

Besides stresses inherent to the illness itself, results from the PA COVID-19 survey revealed a deleterious effect of confinement conditions on PA and significantly increased sitting time. These observations have repercussions that could aid development of PA guidelines to maintain health during COVID-19 and subsequent pandemics. Furthermore, these preliminary results confirm the risk of psychosocial strain during early COVID19 home confinement in 2020. Therefore, to mitigate negative psychosocial effects of home confinement, implementation of national and international initiatives focused on social inclusion is strongly suggested. Increased psychosocial strain triggered by enforced home confinement should encourage stakeholders and policymakers to implement a crisisoriented interdisciplinary intervention to mitigate the negative effects of restrictions and to foster an active and healthy confinement lifestyle (AHCL). Finally, given that present findings are founded on data from a heterogenous population without subsample analysis, further research is warranted to identify subpopulations at greater risk of COVID-19 confinement measures. Identification of such populations would allow for better informed and more targeted mitigation strategies.

Author Contributions: Conceptualization, S.H. and M.S.; methodology, S.H.; software, S.H.; validation, A.S.A.-M., M.S. and A.S.; formal analysis, E.G.B.; investigation, S.H.; resources, M.S.; data curation, R.S.; writing—original draft preparation, S.H.; writing—review and editing, A.S. and L.D.H.; visualization, S.H.; supervision, R.S.; project administration, S.H.; funding acquisition, S.H. All authors have read and agreed to the published version of the manuscript.

Funding: This publication was supported by Qatar University Student Grant QUST-1-CAS-2021-6. The findings achieved herein are solely the responsibility of the authors. 
Institutional Review Board Statement: The study was conducted according to the guidelines of the Declaration of Helsinki, and approved by the Institutional Review Board of QATAR UNIVERSITY (protocol code QU-IRB 1350EA-2020 and date of approval 22 July 2020).

Informed Consent Statement: Informed consent was obtained from all subjects involved in the study.

Data Availability Statement: The raw data supporting the conclusions of this article will be made available by the authors, without undue reservation.

Acknowledgments: We thank our colleagues who provided insight and expertise that greatly assisted the research. We thank all colleagues and people who believed in this initiative and helped to distribute the survey.

Conflicts of Interest: The authors declare no conflict of interest.

\section{References}

1. Hossain, M.M.; Sultana, A.; Purohit, N. Mental health outcomes of quarantine and isolation for infection prevention: A systematic umbrella review of the global evidence. Epidemiol. Health 2020, 42, e2020038. [CrossRef]

2. Ammar, A.; Chtourou, H.; Boukhris, O.; Trabelsi, K.; Masmoudi, L.; Brach, M.; Bouaziz, B.; Bentlage, E.; How, D.; Ahmed, M.; et al. COVID-19 Home Confinement Negatively Impacts Social Participation and Life Satisfaction: A Worldwide Multicenter Study. Int. J. Environ. Res. Public Health 2020, 17, 6237. [CrossRef]

3. Bloch, W.; Halle, M.; Steinacker, J.M. Sport in times of Corona. Ger. J. Sports Med. 2020, 71, 83-84. [CrossRef]

4. Slimani, M.; Paravlic, A.; Mbarek, F.; Bragazzi, N.L.; Tod, D. The relationship between physical activity and quality of life during the confinement induced by COVID-19 Outbreak: A Pilot Study in Tunisia. Front. Psychol. 2020, 11, 1882. [CrossRef] [PubMed]

5. Steinacker, J.M.; Bloch, W.; Halle, M.; Mayer, F.; Meyer, T.; Hirschmüller, A.; Roecker, K.; Wolfarth, B.; Nieß, A.; Reinsberger, C. Merkblatt: Gesundheitssituation für Sportler durch die aktuelle Coronavirus-Pandemie (SARS-CoV-2/COVID-19). Dtsch. Z. Sportmed. 2020, 71, 85-86. [CrossRef]

6. Chen, P.; Mao, L.; Nassis, G.P.; Harmer, P.; Ainsworth, B.E.; Li, F. Coronavirus disease (COVID-19): The need to maintain regular physical activity while taking precautions. J. Sport Health Sci. 2020, 9, 103-104. [CrossRef] [PubMed]

7. Lau, H.M.-C.; Lee, E.W.-C.; Wong, C.N.-C.; Ng, G.Y.-F.; Jones, A.Y.-M.; Hui, D.S.C. The Impact of Severe Acute Respiratory Syndrome on the Physical Profile and Quality of Life. Arch. Phys. Med. Rehabil. 2005, 86, 1134-1140. [CrossRef] [PubMed]

8. Arora, T.; Grey, I. Health behaviour changes during COVID-19 and the potential consequences: A mini-review. J. Health Psychol. 2020, 25, 1155-1163. [CrossRef] [PubMed]

9. Schuch, F.B.; Bulzing, R.A.; Meyer, J.; Vancampfort, D.; Firth, J.; Stubbs, B.; Grabovac, I.; Willeit, P.; Tavares, V.D.; Calegaro, V.C.; et al. Associations of moderate to vigorous physical activity and sedentary behavior with depressive and anxiety symptoms in self-isolating people during the COVID-19 pandemic: A cross-sectional survey in Brazil. Psychiatry Res. 2020, $292,113339$. [CrossRef] [PubMed]

10. Ingram, J.; Maciejewski, G.; Hand, C.J. Changes in Diet, Sleep, and Physical Activity Are Associated With Differences in Negative Mood During COVID-19 Lockdown. Front. Psychol. 2020, 2, 588604. [CrossRef]

11. Ammar, A.; Mueller, P.; Trabelsi, K.; Chtourou, H.; Boukhris, O.; Masmoudi, L.; Bouaziz, B.; Brach, M.; Schmicker, M.; Bentlage, E.; et al. Psychological consequences of COVID-19 home confinement: The ECLB-COVID19 multicenter study. PLoS ONE 2020, 15, e0240204. [CrossRef] [PubMed]

12. Lesser, I.A.; Nienhuis, C.P. The Impact of COVID-19 on Physical Activity Behavior and Well-Being of Canadians. Int. J. Environ. Res. Public Health 2020, 17, 3899. [CrossRef] [PubMed]

13. Brooks, S.K.; Webster, R.K.; Smith, L.E.; Woodland, L.; Wessely, S.; Greenberg, N.; Rubin, G.J. The psychological impact of quarantine and how to reduce it: Rapid review of the evidence. Lancet 2020, 395, 912-920. [CrossRef]

14. Fuentes-García, J.P.; Martínez Patiño, M.J.; Villafaina, S.; Clemente-Suárez, V.J. The effect of COVID-19 confinement in behavioral, psychological, and training patterns of chess players. Front. Psychol. 2020, 11, 1812. [CrossRef] [PubMed]

15. Altena, E.; Baglioni, C.; Espie, C.A.; Ellis, J.; Gavriloff, D.; Holzinger, B.; Schlarb, A.; Frase, L.; Susanna, J.; Riemann, D. Dealing with sleep problems during home confinement due to the COVID-19 outbreak: Practical recommendations from a task force of the European CBT-I Academy. J. Sleep Res. 2020, 4, e13052. [CrossRef]

16. Ning, L.; Niu, J.; Bi, X.; Yang, C.; Liu, Z.; Wu, Q.; Ning, N.; Liang, L.; Liu, A.; Hao, Y.; et al. The impacts of knowledge, risk perception, emotion and information on citizens' protective behaviors during the outbreak of COVID-19: A cross-sectional study in China. BMC Public Health 2020, 20, 1751. [CrossRef] [PubMed]

17. Craig, C.L.; Marshall, A.L.; Sjöström, M.; Bauman, A.E.; Booth, M.L.; Ainsworth, B.E.; Pratt, M.; Ekelund, U.L.; Yngve, A.; Sallis, J.F.; et al. International Physical Activity Questionnaire: 12-Contry Reliability and Validity. Med. Sci. Sports Exerc. 2003, 35, 1381-1395. [CrossRef] [PubMed]

18. Lee, P.H.; Macfarlane, D.J.; Lam, T.H.; Stewart, S.M. Validity of the international physical activity questionnaire short from (PAQ-SF): A systematic review. Int. J. Behav. Nutr. Phys. Act. 2011, 8, 115. [CrossRef] [PubMed]

19. Whitley, E.; Ball, J. Statistics Review 4: Sample Size Calculations. Crit. Care 2002, 6, 335-341. [CrossRef] 
20. Zhang, Y.; Ma, Z.F. Impact of the COVID-19 Pandemic on Mental Health and Quality of Life among Local Residents in Liaoning Province, China: A Cross-Sectional Study. Int. J. Environ. Res. Public Health 2020, 17, 2381. [CrossRef] [PubMed]

21. Diener, E.; Emmons, R.A.; Larsen, R.J.; Griffin, S. The Satisfaction with Life Scale. J. Personal. Assess. 1985, 49, 71-75. [CrossRef] [PubMed]

22. Bortz, J. (Ed.) Basic principle of one-factorial analysis of variance. In Statistics for Social Scientists; Springer: Berlin/Heidelberg, Germany; New York, NY, USA, 1999; pp. 237-249.

23. Hartmann, A.; Herzog, T.; Drinkmann, A. Psychotherapy of bulimia nervosa: What is effective? A meta-analysis. J. Psychosom. Res. 1992, 36, 159-167. [CrossRef]

24. Cohen, J. The effect size. In Statistical Power Analysis for the Behavioural Sciences; Taylor \& Francis: Abingdon, UK, $1988 ;$ pp. 77-83.

25. Richardson, J.T.E. Eta squared and partial eta squared as measures of effect size in educational research. Educ. Res. Rev. 2011, 6, 135-147. [CrossRef]

26. Lee, I.M.; Shiroma, E.J.; Lobelo, F.; Puska, P.; Blair, S.N.; Katzmarzyk, P.T. Lancet Physical Activity Series Working, G. Effect of physical inactivity on major non- communicable diseases worldwide: An analysis of burden of disease and life expectancy. Lancet 2012, 380, 219-229. [CrossRef]

27. Tremblay, M.S.; Warburton, D.E.; Janssen, I.; Paterson, D.H.; Latimer, A.E.; Rhodes, R.E.; Kho, M.E.; Hicks, A.; LeBlanc, A.G.; Zehr, L.; et al. New Canadian physical activity guidelines. Appl. Physiol. Nutr. Metab. 2011, 36, 36-46. [CrossRef] [PubMed]

28. King, A.C.; Powell, K.E.; Kraus, W.E. The US physical activity guidelines advisory committee report-introduction. Med. Sci. Sports Exerc. 2019, 51, 1203-1205. [CrossRef] [PubMed]

29. World Health Organization. Naming The Coronavirus Disease (COVID-19) and The Virus that Causes it; World Health Organization: Geneva, Switzerland, 2020.

30. Ammar, A.; Trabelsi, K.; Brach, M.; Chtourou, H.; Boukhris, O.; Masmoudi, L.; Bouaziz, B.; Bentlage, E.; How, D.; Ahmed, M.; et al. Effects of home confinement on mental health and lifestyle behaviours during the COVID-19 outbreak: Insight from the ECLB-COVID19 multicenter study. Biol. Sport 2020, 38, 37-44.

31. Giustino, V.; Parroco, A.M.; Gennaro, A.; Musumeci, G.; Palma, A.; Battaglia, G. Physical Activity Levels and Related Energy Expenditure during COVID-19 Quarantine among the Sicilian Active Population: A Cross-Sectional Online Survey Study. Sustainability 2020, 12, 4356. [CrossRef]

32. Jukic, I.; Calleja-González, J.; Cos, F.; Cuzzolin, F.; Olmo, J.; Terrados, N.; Njaradi, N.; Sassi, R.; Requena, B.; Milanovic, L.; et al. Strategies and Solutions for Team Sports Athletes in Isolation due to COVID-19. Sports 2020, 8, 56. [CrossRef] [PubMed]

33. Lim, M.A.; Pranata, R. Sports activities during any pandemic lockdown. Ir. J. Med. Sci. 2020, 1-5. [CrossRef]

34. Yeo, T.J. Sport and exercise during and beyond the COVID-19 pandemic. Eur. J. Prev. Cardiol. 2020, 27, 1239-1241. [CrossRef]

35. Myer, G.D.; Faigenbaum, A.D.; Cherny, C.E.; Heidt, R.S.; Hewett, T.E. Did the NFL Lockout expose the Achilles heel of competitive sports? J. Orthop. Sports Phys. Ther. 2011, 41, 702-705. [CrossRef] [PubMed]

36. Pillay, L.; van Rensburg, D.C.; van Rensburg, A.J.; Ramagole, D.A.; Holtzhausen, L.; Dijkstra, H.P.; Cronje, T. Nowhere to hide: The significant impact of coronavirus disease 2019 (COVID-19) measures on elite and semi-elite South African athletes. J. Sci. Med. Sport 2020, 23, 670-679. [CrossRef]

37. Alajmi, J.; Jeremijenko, A.M.; Abraham, J.C.; Alishaq, M.; Concepcion, E.G.; Butt, A.A.; Abou-Samra, A.B. COVID-19 infection among healthcare workers in a national healthcare system: The Qatar experience. Int. J. Infect. Dis. 2020, 100, 386-389. [CrossRef] [PubMed]

38. Al Kuwari, H.M.; Rahim, H.F.A.; Abu-Raddad, L.J.; Abou-Samra, A.B.; Al Kanaani, Z.; Al Khal, A.; Butt, A.A. Epidemiological investigation of the first 5685 cases of SARS-CoV-2 infection in Qatar, 28 February-18 April 2020. BMJ Open 2020, 10, e040428. [CrossRef] [PubMed]

39. Pancani, L.; Marinucci, M.; Aureli, N.; Riva, P. Forced social isolation and mental health: A study on 1006 Italians under COVID-19 quarantine. PsyArXiv 2020, Preprint.

40. Lima, C.K.; de Medeiros Carvalho, P.M.; Lima, I.D.; de Oliveira Nunes, J.V.; Saraiva, J.S.; de Souza, R.I.; da Silva, C.G.; Neto, M.L. The emotional impact of Coronavirus 2019-nCoV (new Coronavirus disease). Psychiatry Res. 2020, 287, 112915. [CrossRef] [PubMed]

41. Zhang, S.X.; Tower, N. Unprecedented disruptions of lives and work-A survey of the health, distress and life satisfaction of working adults in China one month into the COVID-19 outbreak. Psychiatry Res. 2020, 288, 112958. [CrossRef] [PubMed]

42. Lima, C.K.; de Medeiros Carvalho, P.M.; Lima, I.D.; de Oliveira Nunes, J.V.; Saraiva, J.S.; de Souza, R.I.; da Silva, C.G.; Neto, M.L. Effects of COVID-19 home confinement on eating behaviour and physical activity: Results of the ECLB-COVID19 International Online Survey. Nutrients 2020, 12, 1583. [CrossRef]

43. Zahra, D.; Sadatmahalleh, S.J.; Samaneh, Y.; Mahnaz, B.K.; Anoshiravan, K. Influential Factors on Quality of Life in Married Iranian Women during the Covid-19 Pandemic in 2020: A Path Analysis 2020. Available online: https:/ / assets.researchsquare. com/files/rs-27439/v1/605f054d-948c-4f39-99ee-d967d1fb95da.pdf (accessed on 11 August 2020).

44. Guhl, E.; Althouse, A.; Sharbaugh, M.; Pusateri, A.M.; Paasche-Orlow, M.; Magnani, J.W. Association of income and health-related quality of life in atrial fibrillation. Open Heart 2019, 6, e000974. [CrossRef]

45. Mak, W.W.S.; Law, R.W.; Woo, J.; Cheung, F.M.; Lee, D. Social support and psychological adjustment to SARS: The mediating role of self-care self-efficacy. Psychol. Health 2009, 24, 161-174. [CrossRef] [PubMed] 
46. Qi, M.; Li, P.; Moyle, W.; Weeks, B.; Jones, C. Physical Activity, Health-Related Quality of Life, and Stress among the Chinese Adult Population during the COVID-19 Pandemic. Int. J. Environ. Res. Public Health 2020, 17, 6494. [CrossRef] [PubMed]

47. Tsang, C.D. An exploratory study of the impact of stress on quality of life among front-line nursing staff working in respiratory fever admission wards (RFAWs) and general medical wards (GMWs). J. Appl. Phys. Lett. 2008, 92, 191105. 